\title{
Preface: Repair of the Sternum after Median Sternotomy: A Procedure with Complex Problems in Need of a Multidisciplinary Solution
}

Surgical repair of the sternum after open chest surgery is a procedure that involves fixing the divided sides of the sternum. Fixing the bone is a problem that is well known to orthopedic researchers, but sternal closure is a secondary procedure that is performed after the index procedure of cardiac or cardiothoracic vascular surgery. ${ }^{1}$ Healing of the sternum can be compromised by a number of factors including poor fixation that does not effectively hold the two sides together and challenges with high-risk patients including obesity, diabetes, osteoporosis, and chronic lung disease that increase the risk of infection and failure of the sternal closure. ${ }^{1}$ Moreover, the loading situation of the sternum is complicated by the constant cyclic loading during breathing and it can experience bursts of high-energy loading during a cough. Although the traditional closure technique using cerclage wires works in many patients, the complications associated with poor healing of the sternum or infection of the wound can be devastating and associated with a high risk of morbidity and mortality. ${ }^{1}$ Sternal closure is a procedure that needs a multidisciplinary approach to identify solutions appropriate to the patient's needs and condition.

In this issue, Dr. Gandhi provides a three-part critical review of sternal closure with a comprehensive background in sternal anatomy, ${ }^{2}$ assessment of sternal closure techniques, ${ }^{3}$ and clinical decision making. ${ }^{4}$ Part 1 provides comprehensive background with a coverage of sternal anatomy, development, and the role of the sternum in the biomechanics of respiration and coughing. ${ }^{2}$ These issues are critical to an understanding how the physiological forces of breathing and coughing can jeopardize the healing sternum. In part 2, Gandhi examines the role of the sternum in thoracic biomechanics and provides an assessment of current thoracic closure techniques. ${ }^{3}$ In part 3, Gandhi uses the backdrop of "patient-appropriate" medicine to highlight the need for the role of clinical biomedical engineers in defining surgical solutions that are optimized to the patient's needs for this complex problem. ${ }^{4}$ With maturity of a number of technologies, we are on the cusp of full implementation of patient-specific medicine in a number of areas, including optimization of patient flow, ${ }^{5}$ surgical planning, ${ }^{6}$ production of implantable biomaterials, ${ }^{7,8}$ and additive manufacturing of patient-specific implantable devices. ${ }^{9}, 10$ Part 3 of the review speaks to the consideration of patient-specific needs. Gandhi emphasizes that a single "one-size-fits-all" approach ${ }^{11}$ is not suitable for high-risk patients undergoing sternal closure and he uses the term "patient-appropriate medicine" for the problem. Personalized, or patient-specific, medicine has a number of potential benefits for the patient and for reducing treatment and follow-up costs, ${ }^{5}$ but the "just-in-time" manufacturing, tissue engineering, treatment, and patient flow in the health setting requires the involvement of a multidisciplinary team to ensure the maintenance of critical standards. ${ }^{5,7,9,12}$

Treatment of sternal closure, particularly for high-risk patients with complicated bone healing, bone health, and loading conditions, is a challenging problem when examined only from a surgical perspective. A patient-specific approach requires a multidisciplinary approach between clinician and clinical biomedical engineer to ensure identifying optimal closure. To our knowledge, no surgical team has examined the issue in its entirety and Dr. Gandhi brings a unique perspective and provides an integrated approach to applying biomedical engineering to sternal closure in collaboration with surgeons and allied health care professionals.

\section{REFERENCES}

1. Pinotti KF, Cataneo DC, Rodrigues OR, Cataneo AJM. Closure of the sternum with anchoring of the steel wires: Systematic review and meta-analysis. J Thorac Cardiovasc Surg. 2018;156(1):178-86.

2. Gandhi H. Rationale and options for choosing an optimal closure technique for primary mid-sagittal osteo-chondrotomy of the sternum. Part 1: A theoretical and critical 
review of functional anatomy, biomechanics and fracture healing. Crit Rev Biomed Eng. 2019;47(1):1-25.

3. Gandhi H. Rationale and options for choosing an optimal closure technique for primary mid-sagittal osteo-chondrotomy of the sternum. Part 2: A theoretical and critical review of techniques and fixation devices. Crit Rev Biomed Eng. 2019;47(1):27-57.

4. Gandhi H. Rationale and options for choosing an optimal closure technique for primary mid-sagittal osteo-chondrotomy of the sternum. Part-3: Technical decision making based on practice of patient-appropriate medicine. Crit Rev Biomed Eng. 2019;47(1):59-99.

5. Pang Z, Yang G, Khedri R, Zhang YT. Introduction to the special section: Convergence of automation technology, biomedical engineering, and health informatics toward the healthcare 4.0. IEEE Rev Biomed Eng. 2018;249-59.

6. Huff TJ, Ludwig PE, Zuniga JM. The potential for machine learning algorithms to improve and reduce the cost of 3-dimensional printing for surgical planning. Expert Rev Med Devices. 2018;15(5):349-56.

\section{Guest Editor:}

Gregory R. Wohl, PhD, PEng Department of Mechanical Engineering McMaster School of Biomedical Engineering McMaster University Hamilton, Ontario, Canada
7. Aguado BA, Grim JC, Rosales AM, Watson-Capps JJ, Anseth KS. Engineering precision biomaterials for personalized medicine. Sci Transl Med. 201;10(424):eaam8645.

8. Madl CM, Heilshorn SC, Blau HM. Bioengineering strategies to accelerate stem cell therapeutics. Nature. 2018;557(7705):335-42.

9. Trevisan F, Calignano F, Aversa A, Marchese G, Lombardi M, Biamino S, Ugues D, Manfredi D. Additive manufacturing of titanium alloys in the biomedical field: processes, properties and applications. J Appl Biomater Funct Mater. 2018;16(2):57-67.

10. Wong KC. 3D-printed patient-specific applications in orthopedics. Orthop Res Rev. 2016.

11. Veeramachaneni N. The optimal sternal closure technique: Still elusive. J Thorac Cardiovasc Surg. 2018;187.

12. Chhaya MP, Poh PSP, Balmayor ER, Van Griensven M, Schantz JT, Hutmacher DW. Additive manufacturing in biomedical sciences and the need for definitions and norms. Expert Rev Med Devices. 2015;12(5):537-43. 